\title{
Las capacidades para gobernar y la integración europea
} Yehezkel Dror *

\section{Una encrucijada hacia el futuro}

Todos los gobiernos contemporáneos se enfrentan a decisiones cruciales que constituyen, en su esencia, apuestas con la historia sobre trayectorias alternativas hacia el futuro. En los países de la Unión Europea estas disyuntivas se refieren al futuro de la propia Unión, tal y como ponen de manifiesto las cuestiones a discutir en la conferencia intergubernamental de 1996. Ahora bien, tanto en el ámbito europeo como en los ámbitos nacionales y subnacionales, los gobiernos se enfrentan hoy día a decisiones cruciales como las que afectan a los grandes proyectos de infraestructura (por ejemplo, las redes ferroviarias de alta velocidad); a las políticas hacia el Norte de Africa, la emigración y los residentes musulmanes; a las inversiones en ciencia y tecnología; a la lucha contra el desempleo; la reforma del propio Estado; y a muchas más cosas.

Esto supone que las decisiones gubernamentales, si están bien preparadas y formuladas, pueden ejercer una considerable influencia en la forma que adopte la sociedad venidera. Por esta razón, las modas contemporáneas de "reinventar" o "desinventar" el Gobierno y la Administración pública, reduciendo su importancia, son en su mayor parte erróneas. Esto no excluye que la sociedad civil en sus diversas manifestaciones, ya sean mercados, organizaciones no gubernamentales, individuos innovadores y otros actores ajenos al sector público, deba realizar funciones esenciales en "la producción de la sociedad"; tampoco elimina la necesidad de trabajar en la reestructuración de la prestación y gestión de servicios públicos, incuyendo en buena parte su privatización, bajo la idea de que las administraciones públicas deben ser eficaces y eficientes, enjutas y compactas.

Sin embargo, todo ello no debe ocultar un hecho esencial: $a$ pesar de las modas, metáforas y deseos que menosprecian las funciones públicas, el Estado continuará siendo responsable de decisiones cruciales con un enorme poder conformador del futuro. Así ocurre en el caso de la Unión Europea, cuyas instituciones se superponen a las estructuras gubernamentales, "haciendo historia", a través del diseño, consideración, orientación, selección y puesta en práctica de decisiones colectivas democráticas de carácter social.

\section{La Unión Europea, una revolución en la capacidad de gobernar}

Es una peligrosa equivocación pensar que las capacidades de gobernar heredadas del pasado pueden enfrentarse con éxito a los retos presentes y futuros sin cambios radicales. Pero, al mismo tiempo, es evidente que las reformas dirigidas a desarrollar las capacidades gubernamentales necesarias son difíciles de concebir e implantar. Esto genera una tendencia a afirmar que las 
sociedades son ingobemables, haciendo dejación de las responsabilidades públicas y acusando a los "mercados" o a la sociedad civil de problemas que son en su mayor parte una consecuencia de las propias inacapacidades gubernamentales. Tampoco es extraño que, cuando, fruto de la desesperación, los gobiernos se deciden a actuar, lo hagan por caminos que les lleven al fracaso, convirtiéndose en blanco de críticas y acusaciones.

De hecho, puede constatarse empíricamente a lo largo de los países de la Unión Europea que las capacidades para gobernar son dolorosamente insuficientes ${ }^{2}$. Dadas estas incapacidades y sus consecuencias, expresadas en tentativas inútiles y contraproducentes para hacer frente a los problemas o en la simple imposibilidad de satisfacer ciertas aspiraciones sociales, no es extraño que algunos países europeos, entre ellos España, estén pasando por una seria crisis política.

No hay fácil solución a estos problemas que en parte se derivan de transformaciones globales históricas, como las alteraciones en el reparto de poder entre países occidentales y los países de Asia del Este o de los cambios en las tecnologías productivas y sus efectos sobre el mercado de trabajo. Sin embargo, unas capacidades para gobernar más adecuadas permitirían afrontar mejor estos retos y adversidades ${ }^{3}$, ayudando a ponderar las expectativas y demandas sociales.

Paradójicamente, los fracasos de los Estados nacionales se producen en el marco de uno de los esfuerzos más innovadores para crear nuevas capacidades para gobernar, la Unión Europea. Esta constituye un cambio revolucionario en las formas de gobierno, apartándose de trescientos años de dominio del Estado-nación, mediante la creación de una estructura supranacional que provoca la transformación del concepto de soberanía en una nueva entidad política. Desde una perspectiva histórica de largo plazo, la emergencia y evolución de la Unión Europea es un gran éxito de las naciones europeas buscando nuevas capacidades para gobernar que se adecuen a las necesidades del siglo XXI.

No obstante, a pesar del sorprendente éxito que supone la construcción de la Unión Europea, ésta también se enfrenta a sus propias incapacidades en dos ámbitos diferentes: el de la propia Unión y el de sus Estados miembros.

La paradoja es el resultado de retrasos que no son difíciles de entender: la Unión Europea constituye, como decíamos, una revolución en las capacidades de gobernar, pero una revolución inacabada, "un experimento que todavía no ha fracasado", como Martin BuBER caracterizó el movimiento de los kibbutz en Israel, o que no ha llegado a ser un completo éxito. Teniendo en cuenta los retos de un cambio global ${ }^{4}$ a los que se enfrenta la Unión Europea en su conjunto y sus Estados miembros en particular, quedar paralizado en medio del camino antes de lograr una masa crítica de realizaciones, puede equivaler a un fracasos. Para que la integración europea se convierta en una respuesta bistórica capaz de enfrentarse con éxito a los retos de occidente, es preciso continuar avanzando en reformas radicales de su sistema de gobierno, tanto en el ámbito de la Unión, como en el de sus Estados miembros.

Los procesos de rediseño de las capacidades gubernamentales en la Unión y en los Estados miembros se encuentran fuertemente interrelacionados. Sin embargo, en la fase actual de la Unión la mayor parte del peso de desarrollar las capacidades gubernamentales les corresponde a los Estados miembros, tanto como actores del proceso de integración, como en su condición de titulares de importantes poderes de decisión que afectan a su futuro en el seno de la Unión.

Dejando para otra ocasión las reformas necesarias en el ámbito de la propia Unión Europea', este artículo se centra en las mejoras necesarias en la capacidad de gobernar en sus Estados miembros, tanto en lo que afecta a su papel conformador de la propia Unión, como a su condición de actores autónomos dentro de la misma.

\section{Competencias esenciales}

Empleando uno de los pocos conceptos de la literatura de gestión que resulta útil para entender las cuestiones relativas a las capacidades de gobernar, los Estados de la Unión Europea

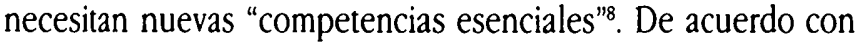
mi trabajo sobre las "capacidades de gobernar" y teniendo en cuenta lo poco que conozco de España ${ }^{10}$, me propongo presentar brevemente una serie de sugerencias para el rediseño del Estado, dirigidas a construir capacidades gubernamentales que sean útiles para afrontar los retos, oportunidades y riesgos de la integración europea en el marco de un proceso de cambio global".

Voy a efectuar recomendaciones "técnicas", evitando cuestiones de naturaleza constitucional o relativas al funcionamiento de los partidos políticos, dado que no conozco suficientemente el sistema español y que, en cualquier caso, no constituyen un tema apropiado para que un "amigo extranjero" ofrezca sus consejos.

Para llegar a alcanzar unas competencias centrales adecuadas para gobernar en relación a la integración europea son necesarias importantes innovaciones, al menos ${ }^{12}$, en las seis direcciones siguientes: dar más peso a la razón de Europa; hacer crecer la confianza en los políticos; incrementar los conocimientos de las elites gubernamentales; orientar la forma de gobernar hacia un profesionalismo innovador; alargar el hori- 
zonte temporal de las políticas públicas, e ilustrar a la opinión pública sobre las decisiones políticas. Voy a detenerme a continuación en cada una de ellas.

\section{Otorgar más peso}

\section{a la "razón de Europa"}

La integración europea plantea un dilema crucial de valores entre la "razón de Estado" y la "razón de Europa" (sin dejar de tener en cuenta, dada su importancia, la "razón de humani$\left.\mathrm{dad}^{\prime}\right)$. A pesar de que en diferentes contextos hay que defender una combinación también diferente entre ambas razones, el proceso de integración europea requiere otorgar más peso y alguna mayor prioridad a la "razón de Europa" sobre la "razón de Estado".

A veces se niega la existencia de este dilema afirmando que el progreso de ambas razones está unido, al menos, en el largo plazo. Es cierto que todos los Estados miembros se benefician de la integración europea, pero también es cierto que a la hora de fijar la posición de un país en decisiones cruciales que afectan al futuro de la Unión, los intereses nacionales pueden entrar en contradicción con el interés europeo. Esto explica la importancia de ponderar el valor que debe otorgarse al progreso de la integración frente a la defensa de los intereses nacionales en el marco de la Unión Europea.

No se trata sólo de una cuestión de valores, sino también de capacidades de comprensión de la realidad. La Unión Europea representa una gran innovación en las formas de gobierno, cuyo desarrollo futuro es indeterminado, mayormente impredecible y en gran parte inconcebible ${ }^{13}$. Por este motivo, la evaluación de las diversas alternativas, en términos de su impacto en el futuro de la Unión Europea, requiere capacidades cognoscitivas tan necesarias como escasas; requiere la habilidad de contemplar tanto las políticas nacionales como las europeas desde la perspectiva de la integración, proporcionando alternativas a los decisores públicos. Esta es una tarea que va más allá de las responsabilidades europeas de los ministerios de asuntos exteriores; hay que pensar en una unidad en los servicios de apoyo al Presidente del Gobierno, que considere las políticas nacionales, así como las posiciones nacionales en las cuestiones europeas y las políticas europeas desde una perspectiva de integración.

Recomendación: una unidad pequeña y compacta en los servicios de apoyo al Presidente del Gobierno deberia encargarse de evaluar las politicas más importantes en términos de la "razón de Europa".

\section{Hacer crecer la confianza en los políticos}

En la medida en que un gobierno debe tomar decisiones costosas en el corto plazo y difíciles en el largo plazo, como todas aquellas que suponen poner por delante la "razón de Europa", frente a la "razón de Estado", resulta más importante la confianza de la población en la clase política. En otras palabras, hacer progresar la integración europea depende en buena parte de la confianza existente en la clase política de los Estados miembros.

Soy consciente de la sensibilidad y complejidad que despierta este tema en los países de la Unión Europea, incluida España. No obstante, reforzar la capacidad de gobernar supone hacer frente a cuestiones sensibles y complejas. Permítaseme, por tanto, ilustrar las medidas dirigidas a este fin, recomendando la elaboración y vigorosa aplicación de códigos éticos para los responsables políticos.

Estos códigos deberían incorporar la necesidad de revelar información personal pertinente, en materia de patrimonio y estado de salud; el compromiso de no enriquecimiento tanto personal como familiar; la prohibición de ofrecer información falsa al Parlamento (lo que es diferente de no facilitar información secreta, que puede estar justificado); sanciones especiales por tolerar actuaciones ilegales; etc.

Los códigos éticos deberían acompañarse de mecanismos institucionales para hacerlos viables, como la reducción en el coste de las campanas electorales o una remuneración suficientemente elevada por el trabajo de los políticos. También resulta necesario agilizar los procesos judiciales para asegurar una rápida investigación de posibles infracciones, seguida de sanciones inmediatas si resultan probadas o de rápidas exoneraciones de responsabilidad en el caso contrario.

Recomendación: deberian adoptarse y bacerse cumplir códigos de conducta para politicos, junto a cambios institucionales que permitan bacer viable su aplicación.

\section{Incrementar}

\section{los conocimientos} de las elites gubernamentales

Resulta menos complicado atender a la necesidad de que las elites gubernamentales conozcan mejor el proceso, los grandes 
temas y las futuras alternativas de la integración europea. Mi propia experiencia en seminarios y contactos con políticos y altos funcionarios de los países de la Unión Europea me permite evaluar la magnitud del esfuerzo requerido, dado que la mayoría de ellos carece de un conocimiento profundo de la integración europea.

Las personas directamente involucradas en las políticas europeas conocen su terreno y algunas de ellas saben mucho. Sin embargo, en la medida en que la integración europea tiene implicaciones para un abanico cada vez mayor de actores y decisiones, es imprescindible que todas las elites gubernamentales, incluyendo el poder judicial, conozcan bien las principales dimensiones de la integración europea ${ }^{14}$.

En consecuencia, deben proporcionarse oportunidades para el aprendizaje, que sean atractivas para los políticos y obligatorias para los funcionarios. Sería deseable la creación de una escuela de ámbito nacional en la que puedan participar las diferentes elites políticas ${ }^{15}$ y gubernamentales para considerar las diferentes cuestiones a las que afecta la integración europea ${ }^{16}$.

Recomendación: deberian darse pasos urgentes para que las elites gubernamentales conozcan mejor el proceso de integración europea. Entre otras cosas, deberian crearse centros nacionales de formación con esta finalidad.

\section{Orientar la forma de gobernar hacia un profesionalismo innovador}

Adquirir conocimientos es relativamente fácil mientras que cambiar la cultura gubernamental es difícil. A pesar de ello, la integración europea requiere cambios profundos en la misma, especialmente en aquellos países de cultura más legalista. Estos cambios tienen tres dimensiones: en primer lugar, desarrollar una cultura más orientada al aprendizaje, más creativa e innovadora, que sea capaz de hacer frente a los cambios que se derivan del proceso de integración europea en el marco de las transformaciones globales; en segundo lugar, cambiar los marcos de referencia de la escala nacional a la europea, de acuerdo con los cambios de valores discutidos anteriormente, $y$, finalmente, hacer evolucionar el énfasis excesivo de los poderes públicos en las disposiciones legales hacia su comportamiento real, sin prescindir de las normas del Estado de Derecho.

Todo ello requiere carreras profesionales diferentes en los niveles más altos de la Administración ${ }^{17}$, combinadas con nuevos esquemas de preparación y formación, nuevas for- mas de liderazgo administrativo e incentivos a la innovación ${ }^{18}$, creando lo que se ha dado en llamar un "profesionalismo innovador".

Recomendación: deberia producirse un cambio en la cultura gubernamental desde su excesiva orientación legalista bacia un mayor profesionalismo innovador, siempre en el marco del Estado de Derecho. Esto requiere, entre otras cosas, nuevos sistemas de formación de los funcionarios públicos, diferentes estructuras de incentivos y nuevas formas de liderazgo administrativo.

\section{Alargar el horizonte temporal de las políticas públicas}

La integración europea constituye una nueva trayectoria hacia el futuro para sus Estados miembros. Es un fenómeno que debe ser considerado en un "tiempo social", lo que supone un espacio temporal mucho más largo que los que se aplican a la mayoría de las políticas públicas ${ }^{19}$. Esta necesidad, sin embargo, se contradice con la configuración actual del sistema democrático, en la que las generaciones futuras no pueden votar y los electores actuales presionan para conseguir resultados inmediatos.

Esta disparidad entre presiones electorales en el corto plazo y procesos sociales de largo plazo como la integración europea, puede convertirse en un "agujero negro", capaz de tragarse la actividad política con efectos pésimos o incluso catastróficos. En mi opinión, la esclavización de los gobiernos a consideraciones de corto plazo y ciclos electorales cortos es una desgracia de las democracias contemporáneas que tiene altos costes y que, de no rectificarse, puede producir efectos fatales.

Frente a esta situación pueden proponerse diversas contramedidas, como las consistentes en otorgar mayor autonomía a los altos funcionarios o establecer organizaciones independientes en su funcionamiento de la lógica política cortoplacista, como los bancos centrales y los tribunales de justicia. Ahora bien, la integración europea necesita mucho más.

Introducir y reforzar consideraciones de largo plazo en la política depende, en buena parte, de un liderazgo creible y orientado hacia el futuro que movilice el apoyo colectivo en tomo a una visión realista a largo plazo ${ }^{2}$. Los códigos de ética pueden ayudar pero, por encima de ello, la posibilidad de disponer de un liderazgo político orientado hacia el futuro que haga políticamente viables las necesidades del largo plazo es una cuestión de suerte y no de "reingeniería en las formas de gobierno".

A pesar de ello, debemos esforzarnos por no depender de accidentes históricos. Deben darse pasos estructurales 
para alargar los horizontes a considerar en la adopción de grandes decisiones. Una adecuada profesionalización de la función pública puede ayudar, así como incrementar el conocimiento de la opinión pública. Pero, al mismo tiempo, son necesarias nuevas estructuras políticas que "institucionalicen" las preocupaciones por el largo plazo en la acción de gobierno.

Puede ser útil crear un "Consejo de Estado", compuesto por personalidades nombradas por un período largo de tiempo por diferentes órganos, como el Rey, las academias científicas y los parlamentos nacionales y regionales. Este órgano tendría derecho a emitir propuestas al gobierno y al Parlamento y la posibilidad de congelar propuestas legislativas hasta la próxima legislatura. Si su composición es la adecuada, este enclave de excelencia protegido de la presión de la actualidad, puede realizar una importante contribución a alargar el horizonte temporal de las políticas públicas, tal y como requiere la integración europea, pero sin quebrar las reglas básicas del sistema democrático.

Recomendación: deberia crearse un "Consejo de Estado", compuesto por personalidades destacadas y con el poder suficiente como para influir en las políticas püblicas.

Una posibilidad más compleja pero muy importante es la de hacer uso de las presiones creadas por la integración europea para introducir consideraciones de largo plazo en la política nacional. Dadas las dificultades de esta última para considerar adecuadamente la orientación de largo plazo, reforzar las capacidades en el ámbito europeo es mucho más necesario. De este modo, la tendencia al corto plazo en el ámbito nacional puede ser contrapesada por una tendencia al largo plazo en el ámbito europeo, permitiendo adoptar a los gobiernos nacionales esta perspectiva, como si viniera forzada por la Unión Europea.

Esto implica tener en cuenta la necesidad de preservar y fortalecer capacidades políticas de largo plazo a la hora de reformar las instituciones europeas. Las extendidas presiones para reducir la autoridad de la Comisión pueden ser contraproducentes en este sentido. Los Estados miembros deberían darse cuenta de estas necesidades y no apresurarse a aplicar ideas nacionales a las instituciones europeas en cuestiones como las relaciones entre el legislativo y el ejecutivo.

Recomendación: fortalecer las capacidades de elaboración de políticas de largo plazo en las instituciones europeas es necesario también para alargar el borizonte temporal de las politicas nacionales. En consecuencia, bay que tener especial cuidado en no debilitar estas capacidades mediante el transplante inadecuado de ideas $e$ instituciones politicas nacionales al ámbito europeo.

\section{Ilustrar a la opinión pública sobre las decisiones políticas}

La única forma posible de combinar la democracia con decisiones nacionales de calidad que consideren, entre otras cosas, un horizonte de largo plazo, es disponer de procesos adecuados de formación de la opinión pública, capaces de enfrentarse adecuadamente tanto a la complejidad como a las necesidades del prever el largo plazo. Alguna literatura entiende que esto puede hacerse directamente por la población ${ }^{21}$, pero yo tiendo a pensar que ésta tiende a situarse por detrás de las nuevas realidades, con la contribución de la mayoría de los medios de comunicación ${ }^{2}$.

Las vicisitudes del Tratado de Maastricht demuestran claramente la ausencia de una opinión pública informada sobre la integración europea. La falta de una acción efectiva en esta dirección en el marco de una visión pluralista es un error manifiesto de la Comisión Europea. Los gobiernos nacionales comparten la culpa, al haber prescindido de "dar un alma ${ }^{\text {"23 a la }}$ integración europea. Los esfuerzos indirectos pueden ayudar como, por ejemplo, proporcionar a los comentaristas de los medios de comunicación la oportunidad de conocer a fondo la integración europea, de modo semejante a lo señalado anteriormente para las escuelas de elites gubernamentales; también puede ayudar la difusión de las deliberaciones del "Consejo de Estado", que se proponía anteriormente. Pero, en cualquier caso, los esfuerzos directos para ilustrar a la opinión pública sobre la integración europea son esenciales, como la difusión de programas televisivos financiados por las instituciones que sean a la vez serios y entretenidos.

Recomendación: ilustrar a la opinión pública es una tarea que los gobiernos deben asumir en el marco del pluralismo democrático. Un paso en esta dirección es la realización de programas televisivos financiados con recursos públicos y dedicados a considerar en profundidad cuestiones relativas a la integración europea.

\section{Las ventajas competitivas de los países}

Asumiendo que se otorga el peso adecuado a la "razón de Europa", el bienestar de los ciudadanos y de las sociedades seguiría siendo la responsabilidad de los gobiernos. Esto supone que, además de progresar en la cooperación, los gobiernos deberían hacer avanzar las ventajas competitivas de sus países en el seno de la Unión Europea. Las capacidades de gobernar 
relacionadas con el proceso de integración europea forman una parte esencial de estas ventajas competitivas ${ }^{24}$.

Adaptar las administraciones a la integración europea, construyendo capacidades y ventajas competitivas, supone muchos cambios de detalle ${ }^{25}$. Pero también hay que pensar que una suma de pequeños cambios no significa necesariamente la transformación necesaria en las capacidades centrales de gobierno. De hecho, si la integración europea es una revolución histórica, son también necesarias revoluciones relacionadas con ella en el seno de los Estados miembros para que tanto la Unión como los Estados puedan prosperar.
Como se ha intentado ilustrar a través de las propuestas presentadas en este artículo, para que las capacidades para gobernar se adecuen a las necesidades y oportunidades que ofrece la integración europea, es necesario rediseñar elementos centrales de nuestro sistema de gobierno y administración. Independientemente del grado de acuerdo o desacuerdo con las propuestas concretas, creo que no puede haber duda sobre la necesidad de innovaciones radicales en las capacidades gubernamentales. Es una tarea dura, pero imprescindible para poder avanzar en la integración europea y beneficiarse de ella.
Arículo iraducido por Koldo ECHEBARRIA ARIZNABARRETA.

- Profesor del Departamento de Ciencia Política de la Universidad Hebrea, de Jerusalem.

' Ver Alain Touraine, Production de la société(París: editions du Seuil), 1973.

${ }^{2}$ Muchos otros paises padecen incapacidades muy superiores, al tiempo que se enfrentan a situaciones mucho más difíciles que la Unión Europea. En este artículo me limito a hablar de ésta última.

${ }^{3}$ Comparar en Yehezkel DroR, Policy Making Under Adversity (New Brunswick, Transaction Books, 1988).

4 Ver Werner WeIDENFELD y Josef JaNNING, eds., Eumpe in Global Change: Strategies and Options for Europe (Gütersloh: Bertelsmann Foundation Publishers), 1993.

' Ver Paul R. Schulman, Large-Scale Policy Making (New York: Elsevier), 1980.

${ }^{6}$ El marco apropiado para considerar estas cuestiones es el ascenso y declive de las civilizaciones y naciones. Algunas de las ideas de Arnold J. TOYNBEE sobre el particular son relevantes.

' Por ejemplo, ver Yehezkel DroR, "Upgrading EC Strategic Choice Capacities", en Futures, 23 (7), 1991: 760-770.

${ }^{8}$ Ver Gary Hamfl y C.K. Prahalad, Competing for the Future (Boston, MA: Harvard Business School), 1994, en especial, el capítulo 10.

- Yehezkel Dror, La capacidad de gobernar: informe al Club de Roma (Barcelona: Galaxia Gutenberg / Círculo de Lectores), 1994.

${ }^{10}$ Aprovecho esta oportunidad para agradecer a los numerosos políticos y funcionarios españoles, así como a mis colegas espanoles del Club de Roma, las oportunidades que me han brindado para conocer un poco de su interesante y agradable país. En particular, me gustaría destacar la imponancia de los tres seminarios sobre capacidades para gobernar y planificación de políticas públicas que he dirigido para altos funcionarios de las administraciones del Estado y las Comunidades Autónomas. En cualquier caso, soy el único responsable de las ideas que de forma tentativa se exponen en este artículo.

"Algunas de estas recomendaciones serian aplicables igualmente sin concurrir la integración europea. No obstante, he seleccionado para este arículo recomendaciones especialmente relevantes en este marco, sin mencionar otras reformas importantes para mejorar las capacidades de gobemar.

12 También son necesarias mejoras radicales en la calidad de los políticos, a los que me refiero de forma pasajera en este ariculo, y cambios significativos en el sistema judicial.
${ }^{13}$ Utilizo la terminología de Carl R. HAUSMAN, A Discourse on Novelty and Creation, (second edition, Albany: State University New York Press), 1984.

14 Dejo a un lado otros conocimientos más mundanos, aunque muy importantes como los idjomas.

is Incluyendo a los comentaristas de los medios de comunicación.

${ }^{16}$ Es sorprendente que la Unión Europea no haya establecido una escuela propia. Estas omisiones en el ámbito europeo hacen aun más necesarias las iniciativas nacionales.

1 Es muy relevante la comparación entre los sistemas administrativos más orientados a la norma y los más orientados a la discrecionalidad profesional, tal y como se efectúa por Bernard S. Silberman, Cages of Reason: The Rise of the Rational State in France, Japan, the United States and Great Britain (Chicago: University of Chicago Press), 1993. La evolución hacia un modelo de discrecionalidad profesional, en una forma revisada y modernizada, es, en mi opinión, esencial para la integración europea, especialmente en las culturas administrativas más legalistas.

${ }^{18}$ Aquí me limito a referirme a algunas de las dimensiones de la necesaria reforma del régimen de empleo público, que se relacionan con la integración europea. No obstante, muchos más cambios son necesarios para construir los sistemas de empleo público del siglo XXI. Ver Yehezkel Dror, "Una elite de alio funcionariado profesional para el siglo XXI", Ekonomiaz: Revista Vasca de Economia, número 26, 1993, pp. 104-119.

19 Aplico la distinción realizada por Fernand BRaUdel entre el "tiempo social" y el tiempo de los acontecimientos ordinarios. El horizonte del tiempo geofísico, al que se refiere también, es relevante a efectos de elaboración de políticas, dado el impacto del comportamiento humano en fenómenos como el clima por ejemplo. Esta necesidad va mucho más lejos de las cuestiones relativas a la integración europea, afectando al futuro de la humanidad como una especie, lo que le hace merecer una consideración separada.

${ }^{20}$ Ver Yehezkel DroR, "Visionary Political Leadership: On Improving a Risky Requisite", International Political Science Review, 9 (1), 1988: 7-22; y los articulos en Gabriel SheFFer, ed., Innovative Leaders in International Politics, (Albany, NY: State University of New York Press), 1993.

"Los que apoyan el populismo o una democracia muy participativa deben hacer suposiciones semejantes implicitas o explícitas (ver Torbjömn TänNsjö, Populist Democracy: A Defence (London: Routledge), 1992. 
${ }^{n}$ Ver, por ejemplo, D. Zow, Democracy and complexity: a Realistic Approacb (Polity Press, Cambridge) 1992.

${ }^{n}$ (Nota del traductor: el término concreto que utliza el autor es soulcraft). Tomo prestada esta palabra tan apropiada como poco convencional de George F. Wu, Statecraft as Soulcraft: Wbat Government Does (New York: Simon \& Schuster), 1983.
* Aquí voy más lejos de las imponantes pero estrechas consideraciones que efectúa Michael R. PORTER, Tbe Competitive Advantage of Nations (London: Macmillan), 1990.

` Ver, por ejemplo, Spyros A. PaPpas, ed., National Administrative Procedures for the Preparation and Implementation of Community Decisions (Maastricht: European Institute of Public Administration), 1995. 


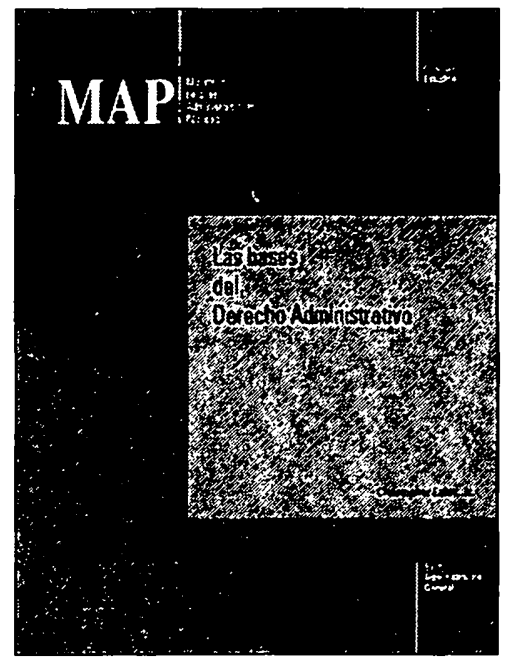

\author{
Las bases del Derecho Administrativo \\ Sabino Cassese \\ MAP/INAP, 1994, 381 págs. 3.000 ptas.
}

El autor, en esta obra, plantea el Derecho Administrativo desde una optica en la que trata de integrar la enorme realidad que supone la actividad administrativa prestacional y las consecuencias que ello tiene en el terreno de la organización, el procedimiento y los recursos financieros de las Administraciones Públicas.

INDICE: La Administración Pública. El Derecho Administrativo. La ciencia del Derecho Administrativo. Las funciones. La organización. El personal. La hacienda. El procedimiento. La Administración transnacional. Ley, Gobierno y Administración Pública. Los jueces y la Administración. Los ciudadanos y la Administración.

\title{
DERECHO ADMINISTRATIVO
}

Reconcebir el control judicial de la Administración Pública Christopher Edley, Jr.

INAP/BOE, 1995, 306 págs. 2.500 ptas.

El objetivo primordial de esta obra es formular una exposición crítica de cómo el Derecho Administrativo determina la acción de gobierno, especialmente a través del control judicial de las acciones de los entes de la Administración Pública.

INDICE: I. La estructura del Derecho Administrativo. Introducción: El problema de la discrecionalidad y la importancia permanente de la teoría de la separación de poderes.-Un ensayo expositivo: la tricotomía constituida por la política, la pericia técnica y la objetividad resolutoria.-Los fallos conceptuales de la tricotomía.-El alcance del control judicial.-П. Reconcebir el Derecho Administrativo. Remedios sin éxito para la inutilidad del Derecho Administrativo.--Un ensayo constructivo: La revisión judicial de tipo de harder-look.-Un ensayo especulativo: De la tricotomía al trío y al control judicial basado en la acción de gobierno concertada.

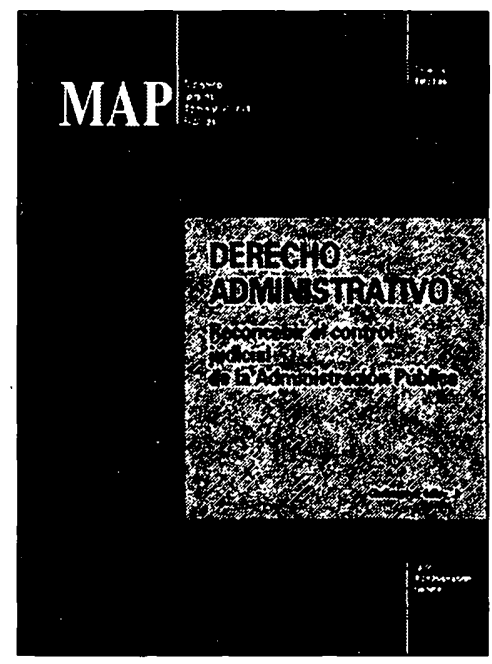

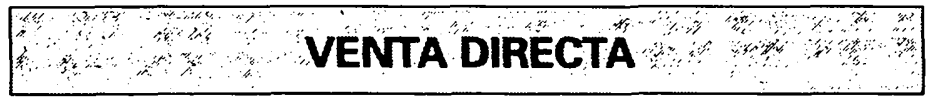

\section{LIBRERIA INAP}

Atocha, 106. 28012 MADRID. Tel. (91) 3493211

\section{LIBRERIA DEL BOE}

Trafalgar, 27. 28010 MADRID. Tel. (91) 53821 00/22 95

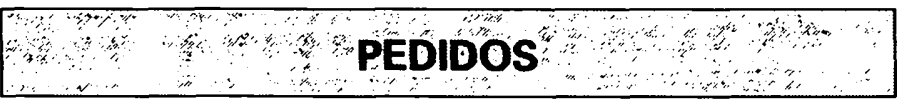

\section{Distribuidor Comercial DOR, S. L.}

Camino de Hormigueras, 124 , planta 6. , naves $\mathrm{E}$ y $\mathrm{F}$ 28031 MADRID.

Tel. (91) 3802875 . Fax (91) 3803440 